ORIGINAL ARTICLE

\title{
Silymarin improves glycemic control through reduction of insulin resistance in newly diagnosed patients of type 2 diabetes mellitus.
}

\author{
Azhar Memon', Shomail Saeed Siddiquiं ${ }^{2}$, Muhammad Atif Ata ${ }^{3}$, Kashif Rasheed Shaikh$^{4}$, Umair Ali Soomro5, Shumaila Shaikh ${ }^{6}$
}

Article Citation: Memon A, Siddiqui SS, Ata MA, Shaikh KR, Soomro UA, Shaikh S. Silymarin improves glycemic control through reduction of insulin resistance in newly diagnosed patients of type 2 diabetes mellitus. Professional Med J 2022; 29(3):362-366.

https://doi.org/10.29309/TPMJ/2022.29.03.6477

\begin{abstract}
Objective: To study, analyse and compare the effects of Silymarin on glycemic control and insulin resistance in newly diagnosed type 2 diaebtes mellitus (T2DM) subjects. Study Design: Observational study. Setting: Suleman Roshan Medical College Hospital. Period: March 2019 to February 2020. Material \& Methods: A sample of 200 newly diagnosed cases of T2DM were recruited accroding to inclusion criteria selected by non-probability convenient sampling. Subjects were divided into 2 groups; OHA- oral hypoglycemia agent and OHA+ Sillymarin (200 mg). Baseline fasting (FBG) and random blood glucose (RBG), glycated HbA1 (A1C), fasting insulin (FI) and insulin resitance (HOMA-IR) were detected. Silymarin therapy was continued for 3 months. Study variables were analysed after 3 months. Data was analyzed on SPSS (ver.19) at $95 \%$ confidence interval $(P \leq 0.05)$ considered statistially significant. Results: Age of diabetics taking oral hypoglycemic agents $(\mathrm{OHA})$ and diaebtic taking $\mathrm{OHA}+$ Silymarin supplementation was noted as $50.3 \pm 13.349 .9 \pm 14.5$ years $(P=0.91) .3$ months Silymarin supplementation improves the fasting blood glucose, random blood glucose, glycated hemoglobin A1 (A1C), fasting insulin and insulin resistance (HOMA-IR) $(P=0.0001)$. Conclusion: Silymarin improves blood glucose levels in type 2 diabetics that is mediated through reduction of insulin resistance.
\end{abstract}

Key words: $\quad$ Glycemic Control, Insulin Resistance, Silymarin, Type 2 Diabetics.

\section{INTRODUCTION}

The incidence and prevalence of type 2 diabetes mellitus (T2DM) is seriously increasing throughout the World. DM is a glucose metabolic disorder of chronic hyperglycemia caused by insulin deficiency. This insulin deficiency may be absolute or relative. Absolute insulin deficiency is observed in the type $1 \mathrm{DM}$ while relative deficiency is seen T2DM. Relative insulin deficiency is caused by receptor insensitivity or post receptor signalling defects. T2DM is characterized by chronic hyperglycemia that damages various tissues and organs especially in insulin independent cells such as the retina, nerve fibers, kidenys, etc. Hence, the long term complications of DM affect the eyes, nerves, blood vessles, heart and kidneys frequently., World prevalence of DM was 382 million in 2013 as reported by the International Diabetes Federation
(IDF), this accounts $8.3 \%$ of total World's population. Prevalence of DM will increase to 592 millions by the 2035 that means 3 new cases each 10 seconds. ${ }^{1,2}$ The hallmark of T2DM is a progressive decline of $\beta$-cell function of pancreas and reduced insulin sensitivity of target organs. This eventually results in chronic hyperglycemia, a hallmark feature of T2DM. Insulin resistance is aggravated by the oxidative stress, inflammation and inflammatory mediators.,4 Cardiovascular system is the most often affected system in DM due to severe dyslipidemia. Hyperlipidemia and dyslipidemia result from metabolic defects caused by insulin deficiency. Lipid peroxidation increases the risk of cardiovascular diseases. Current care of DM includes the pharmacological and non-pharmacological interventions. Caloric restrictions and physical exercise are the life style modifications helpful for glycemic control.
1. MBBS, M.D, Assistant Professor Medicine, Suleman Roshan Medical College, Tando Adam, Sindh, Pakistan. 2. MBBS, M.Phil, Assistant Professor Pathology, Indus Medical College Tando Muhammad Khan, Sindh, Pakistan 3. MBBS, M.Phil, Professor Biochemistry, Suleman Roshan Medical College, Tando Adam, Sindh, Pakistan.

4. MBBS, M.Phil, Associate Professor Pharmacology, Suleman Roshan Medical College, Tando Adam, Sindh, Pakistan

5. MBBS, M.Phil, Assistant Professor Hematology, Indus Medical College Tando Muhammad Khan, Sindh, Pakistan.

6. MBBS, M.Phil, Assistant Professor Biochemistry, Khairpur Medical College, Khairpur Mirs, Sindh, Pakistan.

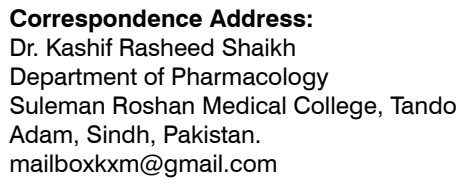


Pharmacological intervensions include the; oral hypoglycemic agents and the insulin therapy. Depsite much efficacy of current drug therapy of $\mathrm{DM}$, the adverse drug reactions (ADRs) is problematic, hence natural remedies have been analyzed of their anti - diabetic efficacy. ${ }^{3,4}$ Natural remedies have been reproted of their beneficial antioxidants effects that neutralize the oxidative stress of chronic hyperglycemia. Silymarin ${ }^{5,6}$ is one of the vastly researched herbal agents that are currently reproted for various diseases. Silymarin is derived from milk thistle plant (Silybum marianum (L.). It is rich in polyphenolic flavonolignan that show potential of anti-oxidant properties. Silymarin includes the 4 flavonolignan isomers and oneflavonoid. Flavonolignans include the; iso- silybin, silybin, silychristin and silydianin and flavonoid include the taxifolin. ${ }^{5,6}$ Silymarin is known of its hypolipidemic, hypoglycemic, antiatherosclerosis, anti-osteoporosis, renoprotective and anti-cancer activities. Silymarin has been used in sepsis, burn therapy and Alzheimer disease..$^{5,6}$

The present study was planned to analyse and compare the blood glucose regulating efficacy and anti-insulin resistance properties of Silymarin in type 2 diabetic subjects presenting at our tertiary care hospital.

\section{MATERIAL \& METHODS}

The present observational study was conducted at the Department of Medicine and Pharmacology, Suleman Roshan Medical College Hospital from March 2019 to February 2020. Study protocol was presented and was approved by the research ethics committee. Participants were interviewed of the purpose of study and were educated to adhere to the drug therapy. Participants were handled in coformation to the Declaration of Helsinki (1975).

A sample of 200 newly diagnosed cases of T2DM were recruited accroding to inclusion criteria selected by non-probability convenient sampling. Subjects were divided into 2 groups; oral hypoglycemia agent $(\mathrm{OHA})$ (glimepride+metformin) and $\mathrm{OHA}$ (glimepride+metformin) + Sillymarin (200 mg bid). DM was dignosed according to the criteria of ADA (American Diabetes Association). Newly diagnosed DM, age $40-60$ years with suboptimal glycemic control were inlcuded. Diet and physical exercise were continued. Exclusion criteria were; chronic liver disease, ischemic heart disease, renal insufficience (SCr $>2 \mathrm{mg} / \mathrm{dl})$, severe heart dysfunction (NYHA class III or more), cerebral stroke, psychiatric disease, smoking, steroid use and severe infections. Patients taking multivitamin, multi-mineral pills were also excluded. Pregnancy and breastfeeding female were strictly excluded. Verbal consent was taken from the participants. The subjects were informed the inclusion to study protocol is on voluntary basis, it will cause no harm or monetorial expenses and they can leave at any time if feeling worried or without telling the reason and this will not affect their diabetic therapy. At baseline, all patients underwent thorough history taking and clinical examination. Blood samples were collected to measure the following parameters at baseline and at end of treatment and used to evaluate the treatment outcomes, baseline fasting (FBG) and random blood glucose (RBG), glycated $\mathrm{HbA1}$ $(\mathrm{A} 1 \mathrm{C})$, fasting insulin $(\mathrm{FI})$ and insulin resitance (HOMA-IR) were detected. Silymarin therapy was continued for 3 months. Fasting (FBG) and random blood glucose (RBG), glycated $\mathrm{HbA1}$ $(\mathrm{A} 1 \mathrm{C})$, fasting insulin $(\mathrm{FI})$ and insulin resitance (HOMA-IR) were repeated after $3^{\text {rd }}$ month drug therapy.

Blood glucose was estimated by hexokinase method, A1C by TINIA - colorimetric method, and fasting inuslin (FI) by ELISA assay kit. Homeostasis Model Assessment - Insulin Resistance (HOMA-IR) was calculated from FBG and $\mathrm{FI}$ by formula as; HOMA-IR = Fasting Blood Glucose $(\mathrm{mg} / \mathrm{dL}) \times$ Fasting Insulin $(\mu \mathrm{U} / \mathrm{m}) / 405 .^{7}$ Baseline and $3^{\text {rd }}$ month laboratory findings were noted in a pre - structured proforma. Data was entered in a Microsoft Excel Sheet (ver. 13.0). Data was kept in lockers to maintain the confidentiality of personal patient records. Data was copied to the SPSS (version 19.0). Data was analysed by Student $t$ - test (Paired sample $t$ - test). 95\% confidence interval $(\mathrm{Cl})$ was taken statistially significant $(P \leq 0.05)$. 


\section{RESULTS}

Age of Diabetics taking oral hypoglycemic agents $(\mathrm{OHA})$ and diaebtic taking $\mathrm{OHA}+$ Silymarin supplementation (SS) was noted as $50.3 \pm 13.3$ $49.9 \pm 14.5$ years $(P=0.91)$. Baseline fasting blood glucose (FBG), random blood glucsoe (RBG), glycated hemoglobin $A 1$ (A1C), fasting insulin (FI) and insulin resistance (HOMA-IR) were noted at baseline (Table-I to V). After 3 months Silymarin supplementation (SS) the FBG, RBG, A1C, Fl and HOMA-IR were rechecked and found improved as shown in Table-I to V. Differences were found statistically significant $(P=0.0001)$.

\section{DISCUSSION}

The present observational study was conducted at tertiary hospital of Suleman Roshan Medical College Hospital Tando Adam. Age of Diabetics taking oral hypoglycemic agents (OHA) and diaebtic taking $\mathrm{OHA}+$ Silymarin supplementation (SS) was noted as $50.3 \pm 13.349 .9 \pm 14.5$ years $(P=0.91)$. The findings are in agreement with previous studies. ${ }^{8,9}$ We found 3 months Silymarin supplementation (SS) reduced the FBG, RBG, $A 1 C, F I$ and HOMA-IR $(P=0.0001)$. The findings are concordant with previous studies. ${ }^{9,10}$

\begin{tabular}{|l|c|c|c|}
\hline & Mean & Std. Deviation & P-Value \\
\hline Baseline - Diabetic (OHA) & 319.70 & 23.43 & 0.91 \\
\hline Baseline - Diabetic (OHA+Silymarin) & 315.40 & 30.72 & 0.0001 \\
\hline 3 month - Dibaetic (OHA) & 243.25 & 41.28 & 0.28 \\
\hline 3 months Diabetic (OHA+Silymarin) & 188.95 & 55.36 & \\
\hline
\end{tabular}

Table-l. Fasting blood glucose $(\mathrm{mg} / \mathrm{dl})$ in study groups.

\begin{tabular}{|l|c|c|c|}
\hline & Mean & Std. Deviation & P-Value \\
\hline Baseline - Diabetic (OHA) & 319.70 & 23.43 & 0.91 \\
\hline Baseline - Diabetic (OHA+Silymarin) & 315.40 & 30.72 & \multirow{2}{*}{0.0001} \\
\hline 3 month - Dibaetic (OHA) & 243.25 & 41.28 & 55.36 \\
\hline
\end{tabular}

Table-II. Random blood glucose $(\mathrm{mg} / \mathrm{dl})$ in study groups.

\begin{tabular}{|l|c|c|c|}
\hline & Mean & Std. Deviation & P-Value \\
\hline Baseline - Diabetic (OHA) & 8.66 & 0.72 & \multirow{2}{*}{0.91} \\
\hline Baseline - Diabetic (OHA+Silymarin) & 8.50 & 0.67 & \multirow{2}{*}{0.0001} \\
\hline 3 month - Dibaetic (OHA) & 7.09 & 0.78 & 0.50 \\
\hline 3 months Diabetic (OHA+Silymarin) & 6.49 & \\
\hline
\end{tabular}

Table-III. A1C (\%) in study groups.

\begin{tabular}{|l|c|c|c|}
\hline & Mean & Std. Deviation & P-Value \\
\hline Baseline - Diabetic (OHA) & 16.28 & 1.07 & 0.91 \\
\hline Baseline - Diabetic (OHA+Silymarin) & 16.06 & 1.20 & 0.0001 \\
\hline 3 month - Dibaetic (OHA) & 14.32 & 1.14 & 0.86 \\
\hline 3 months Diabetic (OHA+Silymarin) & 13.71 & 0.86 & \\
\hline
\end{tabular}

Table-IV. Fasting insulin $(\mu \mathrm{U} / \mathrm{L})$ in study groups.

\begin{tabular}{|l|c|c|c|}
\hline & Mean & Std. Deviation & P-Value \\
\hline Baseline - Diabetic (OHA) & 4.58 & 1.05 & 0.91 \\
\hline Baseline - Diabetic (OHA+Silymarin) & 4.06 & 0.96 & \multirow{2}{*}{0.0001} \\
\hline 3 month - Dibaetic (OHA) & 1.82 & 0.71 & 0.21 \\
\hline 3 months Diabetic (OHA+Silymarin) & 1.35 & 0.21 & \\
\hline
\end{tabular}

Table-V. Insulin resistance (HOMA-IR) in study groups. 
Silymarin proved an excellent glucose regulator by decreasing the insulin resistance. Several mechanisms of anti-diabetic activity of Silymarin have been proposed hence it has attracted the researchers for the diabetic therapy and its complications. ${ }^{8}$ Previous studies ${ }^{10-12}$ have documented the therapeutic potential of Silymarin and its derivatives in both in-vivo and in-vitro studies. Silymarin shows anti-oxidant, anti-inflammatory, anti-gluconeogenesis and membrane stabilizing potential. Insulin gene expression and $\beta$-cell proliferation is documented. Silymarin exerts the peroxisome proliferatoractivated receptor-y (PPARY) agonist like activity. Above findings ${ }^{10-12}$ make silymarin ideal of its anti-diabetic potential. Positive effect on glycemic control and insulin resistance reducing potential of silymarin is documented in our present study and the findings are in line with above studies. ${ }^{13,14}$

In present study, the silymarin significantly decreased FBG, RBG, A1C, FI and HOMA-IR compared to control group. The findings of present study are of clinical significance as the burden of DM is increasing in the country and silymarin is herbal agent with less side effects that may be exploited of its anti-diabetic activity. Anti-diabetic potential of Silymarin is supported by another previous study ${ }^{15}$ that supplemnted $200 \mathrm{mg}$ dose thrice daily for T2DM subjects for 120 days along with glibenclamide and reported optimal glycemic control. A1C was found low compared to baseline by that study, and finding is highly concordant to the present study. Another previous study ${ }^{16}$ used $600 \mathrm{mg}$ silymarin daily in diabetics with alcoholic cirrhosis, and found reduciton in FBG, A1C and Fasting Insulin (FI). It has been reported that the polyphenolic compounds of silymarin also modify the glycemic and lipid profile of type 2 diabetics. ${ }^{15,17}$ Anti diabetic activity of silymarin has been reported by another previous study ${ }^{18}$ that used $140 \mathrm{mg}$ silymarin three times a day for 45 days in type 2 diabetics and found positive effects on glycemic and lipid profile. Mitigation of insulin resistance is consistent with other previous studies. ${ }^{18-20}$ Reduction of insulin resistance (HOMA-IR) and fasting insulin (FI) are findings of great clinical significance. The findings of present study are supported by above reviewed literatrue however further studies are warranted with large sample size in indigenous population to reach to a stronger conclusion. Silymarin shows positive effect on the glycemic control and insulin resistance that needs further elaboration in large prospective studies in future.

\section{CONCLUSION}

Silymarin has potential of improving blood glucose levels in type 2 diabetics that is mediated through reduction of insulin resistance. As the insluin resistance is major problem in type 2 diabetics hence silymarin may be prescribed as a natural remedy to overcome this problem.

Copyright $\odot 21$ May, 2021.

\section{REFERENCES}

1. Artasensi A, Pedretti A, Vistolli G, Fumagalli L. Type 2 diabetes mellitus: $A$ review of multi-target drugs. Molecules 2020; 25(8): 1987.doi: 10.3390/ molecules25081987.

2. Basit A, Fawwad A, Qureshi H, Shera AS. Prevalence of diabetes, pre-diabetes and associated risk factors: Second national diabetes survey of Pakistan (NDSP), 2016-2017. BMJ Open 2018; 8:020961.

3. Pham Tb, Nhuyen TT, Truong HT, Trinh CH, Du HNT, Ngo TT, et al. Effects of Diabetic complications on health related quality of life impairment in Vietnamese patients with type 2 diabetes. J Diabet Res 2020; Article ID 4360804 | https://doi.org/10.1155/2020/4360804.

4. Memon IA, Almani SA, Shaikh TZ, Ujjan I, Kazi N, Khoharo HK. Berberine mitigates insulin resistance in newly diagnosed type 2 diabetics. Int' I J Med Sci Clin Inv 2017; 4(1): 2566-2572.

5. Delmas D. Silymarin and derivatives: From biosynthesis to health benefits. Molecules 2020; 25(10): 2415.

6. Jamalian M, Mahmodiyeh B, Saveiee S, Solhi H. Investigating the impact of silymarin on liver oxidative injury. J Family Med Prim Care 2020; 9:170711.

7. Matthews DR, Hosker JP, Rudenski AS, Naylor BA, Treacher DF, Turner RC. Homeostasis model assessment: Insulin resistance and beta-cell function from fasting plasma glucose and insulin concentrations in man. Diabetologia $1985 \mathrm{Jul}$; 28(7):412-9. 
8. Elgarf AT, Mahdy MM, Sabri NA. Effect of silymarin supplementation on glycemic control, lipid profile and insulin resistance in patients with type 2 diabetes mellitus. Int'l J Adv Res 2015; 3 (12): 812 - 21.

9. Tajmohammadi A, Razavi BM, Hosseinzadeh $\mathrm{H}$. Silybum marianum (milk this-tle) and its main constituent, silymarin, as a potential therapeutic plant inmetabolic syndrome: A review. Phyther Res 2018;32(10):1933-49.

10. Detaille D, Sanchez C, Sanz N, Lopez-Novoa JM, Leverve $X$, El-Mir MY. Interrelation between the inhibition of glycolytic flux by silibinin and the lowering of mitochondrial ROS production in perifused rat hepatocytes. Life Sci 2008; 82:1070-1076.

11. Pferschy-Wenzig EM, Atanasov AG, Malainer C, Noha $S M$, Kunert $O$, Schuster $D$, et al. Identification of isosilybin a from milk thistle seeds as an agonist of peroxisome proliferator-activated receptor gamma. J Nat Prod 2014; 77:842-847.

12. Soto C, Raya L, Juarez J, Perez J, Gonzalez I. Effect of Silymarin in Pdx-1 expression and the proliferation of pancreatic beta-cells in a pancreatectomy model. Phytomedicine 2014; 21:233-239.

13. MacDonald-Ramos K, Layla Michánb L, MartínezIbarraa A, Cerbóna M. Silymarin is an ally against insulin resistance: A review. Ann Hepatol 2020:1-10.

14. Mohammadi SM, Kianbakht S, Rezazadeh Sh, Ziaee $M$, Fallah Huseini $H$. Clinical efficacy of Silybum marianum seed extract in treatment of type 2 diabetes mellitus and non-alcoholic fatty liver disease: $A$ narrative review. J Med Plants 2020; 19(73): 12-26.
15. Huseini HF, Larijani $B$, Heshmat R, Fakhrzadeh $H$, Radjabipour B, Toliat T, Raza M. 2006. The efficacy of Silybum marianum (L.) Gaertn. (silymarin) in the treatment of type II diabetes: A randomized, doubleblind, placebo-controlled, clinical trial. Phytother Res 2006:1036-1039.

16. Velussi M, Cernigoi AM, De Monte A, Dapas F, Caffau C, Zilli M. 1997. Long-term (12 months) treatment with an anti-oxidant drug (silymarin) is effective on hyperinsulinemia, exogenous insulin need and malondialdehyde levels in cirrhotic diabetic patients. J Hepatol 1997; 26:871-879.

17. Sajedianfard J, Behroozi Z, Nazifi S. The effects of a hydroalcoholic extract of silymarin on serum lipids profiles in streptozotocin induced diabetic rats. Comparative Clin Pathol 2014; 23:779-784.

18. Ebrahimpour Koujan S, Gargari BP, Mobasseri $M$, Valizadeh $H$, Asghari-Jafarabadi $M$. Effects of Silybum marianum (L.) Gaertn. (silymarin) extract supplementation on antioxidant status and hsCRP in patients with type 2 diabetes mellitus: A randomized, triple-blind, placebo-controlled clinical trial. Phytomedicine 2015; 22:290-296.

19. Yao J, Zhi M, Gao X, Hu P, Li C, Yang X. Effect and the probable mechanisms ofsilibinin in regulating insulin resistance in the liver of rats with non-alcoholicfatty liver. Braz J Med Biol Res 2013; 46(3):1-9.

20. Zhang Y, Hai J, Cao M, Zhang Y, Pei S, Wang J, et al. Silibinin ameliorates steato-sis and insulin resistance during non-alcoholic fatty liver disease developmentpartly through targeting IRS-1/PI3K/Akt pathway. Int Immunopharmacol 2013; 17(3):714-20.

\begin{tabular}{|c|c|c|c|}
\hline \multicolumn{4}{|c|}{ AUTHORSHIP AND CONTRIBUTION DECLARATION } \\
\hline No. & Author(s) Full Name & Contribution to the paper & Author(s) Signature \\
\hline 1 & $\begin{array}{l}\text { Azhar Memon } \\
\text { Shomail Saeed Siddiqui }\end{array}$ & $\begin{array}{l}\text { Data collection, drug dose, } \\
\text { concept, Data analysis. } \\
\text { Concept, Drug arrangement, } \\
\text { Performa. }\end{array}$ & \\
\hline 3 & Muhammad Atif Ata & $\begin{array}{l}\text { Study setting, arrangement pt } \\
\text { data. }\end{array}$ & \\
\hline 4 & Kashif Rasheed Shaikh & $\begin{array}{l}\text { Pt counseling and blood } \\
\text { sample Procedure. }\end{array}$ & \\
\hline 5 & Umair Ali Soomro & $\begin{array}{l}\text { Hemotological examination of } \\
\text { blood sample. }\end{array}$ & \\
\hline 6 & Shumaila Shaikh & $\begin{array}{l}\text { Manuscript writing, Data } \\
\text { analysis, Proof reading. }\end{array}$ & \\
\hline
\end{tabular}

